\title{
Quantitation of ligand is critical for ligand-dependent MET signalling activation and determines MET-targeted therapeutic response in gastric cancer
}

\author{
Seokhwi Kim ${ }^{1}$ Ji Mi Ahn' ${ }^{1}$ Won Jung Bae ${ }^{1}$ Jae Ho Han ${ }^{1}$. Dakeun Lee ${ }^{1}$ (i) \\ Received: 24 July 2020 / Accepted: 26 October 2020 / Published online: 9 November 2020 \\ (c) The International Gastric Cancer Association and The Japanese Gastric Cancer Association 2020
}

\begin{abstract}
Background Despite the promising preclinical antitumor activity of MET-targeting therapies, most clinical trials have failed. We introduced a new concept of quantitation of stroma-induced hepatocyte growth factor (HGF) to assess the actual MET signalling activity in gastric cancer (GC).

Methods We treated serially diluted HGF and conditioned media (CM) from cancer-associated fibroblasts (CAFs) on low MET-expressing cancer cells and investigated the phenotypical and signalling changes. Stromal proportion and MET expression in GC samples were assessed, and gene set enrichment analysis (GSEA) from the public database was performed. The antitumor effect of anti-MET treatment was examined, especially when cancer cells were activated in a ligand-dependent manner.

Results Relatively high doses of HGF or high-concentrated CM fully activated MET signalling cascades and promoted cell proliferation/invasion. High stromal proportion denoted worse patient survival in MET-positive GCs than in MET-negative ones. GSEA showed that the gene sets regarding proliferation, migration, and CAF as well as MET pathway signature were enriched in simultaneously MET- and HGF-positive samples. Sufficient ligand-dependent MET signalling activation increased the sensitivity to crizotinib.

Conclusions We conclude that patients whose tumours have a high stromal proportion and at least low MET expression may benefit more from MET-targeted therapies.
\end{abstract}

Keywords Gastric cancer $\cdot$ Hepatocyte growth factor $\cdot$ MET $\cdot$ Target therapy $\cdot$ Tumour microenvironment

\section{Background}

The met protooncogene (MET) encodes the receptor tyrosine kinase c-MET (or MET), whereas hepatocyte growth factor (HGF) is the only known ligand. The binding of HGF to MET results in receptor homodimerization with subsequent autophosphorylation of tyrosine residues in its carboxylterminal domain and activation of downstream cascades

Electronic supplementary material The online version of this article (https://doi.org/10.1007/s10120-020-01139-4) contains supplementary material, which is available to authorized users.

Dakeun Lee

dakeun@gmail.com

1 Department of Pathology, Ajou University School of Medicine, 164, Worldcup-ro, Yeongtong-gu, Suwon-si, Gyeonggi-do 16499, Republic of Korea such as mitogen-activated protein kinase (MAPK), phosphoinositide 3-kinase (PI3K), and Rac1-Cdc42 signalling [1]. Therefore, the activation of MET signalling results in phenotypes such as cell proliferation, cell motility, and cell cycle progression [2]. Although MET signalling is important in the control of tissue homeostasis such as embryogenesis and wound healing under physiologic conditions [3], aberrant activations of MET signalling such as gene amplification or protein overexpression have also been found in various human malignancies including gastric cancer (GC) [4].

Clinically, it is known that MET-amplified GCs constitute only a small subgroup (2.2-7.1\%) but are very aggressive [5-7]. Providing MET inhibition represents a rational therapeutic strategy for this group. In several preclinical studies, selective MET inhibitors conferred extraordinarily to apoptosis induction in cancer cell lines or xenografts harbouring MET amplification [8, 9]. However, there was no effect on those cells or xenografts negative for this genetic alteration 
$[8,9]$. These findings suggest that $M E T$-amplified cancer cells are genuinely addicted to oncogenic MET signalling. Accordingly, in some clinical trials, the promising antitumor activity of MET-targeted therapy has been demonstrated in patients with $M E T$-amplified cancer [6, 10].

Besides gene amplification, MET signalling can also be activated by binding to its ligand, HGF. However, there is no specific biomarker that can reflect HGF-dependent MET activation in cancer tissue. Thus, clinical trials that assessed drugs targeting the HGF-MET axis could not help but to enrol MET-(over)expressing cancer patients. In GC, MET expression is far more frequent than amplification with a wide reported range, from 24 to 74\% [11-14]. Although MET overexpression, without MET amplification, has been regarded to be mostly attributed to $M E T$ transcriptional upregulation in GC, the relationship between MET overexpression and the dependence of a tumour on MET signalling still remains unclear [4]. Therefore, it is not surprising that many of these clinical trials have failed to show survival benefit [15-17]. These previous experiences suggest that a more appropriately defined group of patients should be selected in the future. This needs at least a proof of concept that verifies the existence and implication of HGF-dependent MET activation in patients with GC and that ligand-dependent MET signalling activation can be a potential therapeutic vulnerability that can be exploited using specific drugs targeting the HGF-MET axis.

To assess the ligand-dependent MET signalling activation, we introduced a new concept of quantitation of stromainduced HGF. Using this, we estimated the required amount of HGF in the tumour microenvironment and identified the minimal intensity of MET expression in cancer cells to be fully activated in an HGF-dependent manner. Through these processes, we aimed to retain the proof of concept on the significance of ligand-dependent MET activation in patients with GC and to eventually find an appropriate patient group who can potentially benefit from MET-targeted therapy.

\section{Methods}

\section{Human GC samples and clinical data}

We collected formalin-fixed, paraffin-embedded (FFPE) tissues from 272 patients with advanced GC who underwent surgery between January 2005 and December 2006 at the Ajou University Hospital, Republic of Korea. Clinical data were retrieved from patient medical records. Patients were excluded if they had been treated with preoperative chemotherapy or radiotherapy. Patients with distant metastasis at the time of surgery were also excluded. Pathological stages were determined based on the American Joint Committee on Cancer (AJCC), 7th edition. The clinicopathological information is summarised in Table S1. Recurrence-free survival time was defined as the interval between the date of surgery and the first recurrence or death.

\section{Cells}

Human GC cell lines, Kato III, MKN1, MKN45, MKN74, SNU1, SNU216, SNU668, AGS, NCI-N87, GCIY, and NUGC-4 were purchased from the Korean cell bank (KCLB) and RIKEN cell bank and maintained in RPMI 1640 (Hyclone, South Lagan, UT, USA) supplemented with $10 \%$ foetal bovine serum (Hyclone) and 1\% penicillin and streptomycin (Gibco, Thermo Fisher Scientific, Inc., Waltham, MA, USA) at $37{ }^{\circ} \mathrm{C}$ under a humidified atmosphere containing $5 \% \mathrm{CO}_{2}$. All cell lines were routinely tested for mycoplasma. Cancer-associated fibroblasts (CAFs) were isolated from GC specimens, as described in our previous study [18]. CAFs of less than eight passages were used for the experiments to minimise the alteration from the original phenotype.

\section{Antibodies and reagents}

The following antibodies were used for analysing the MET downstream cascade: MET, phospho (p)-MET, AKT, p-AKT, ERK, p-ERK (1:1000-3000; Cell signalling technology, Danvers, MA, USA), and GAPDH (1:10,000; Novus Biologicals, Littleton, CO, USA). Anti-HGF neutralising antibody and control immunoglobulin G (R\&D Systems Inc., Minneapolis, MN, USA) were utilised for the HGFblocking experiments. The following reagents were used to treat the cancer cells: recombinant human HGF (Peprotech, Rocky Hill, NJ, USA) and crizotinib (Cell signalling technology).

\section{Quantitative real-time polymerase chain reaction (qRT-PCR)}

Total RNA was isolated using TRIzol reagent (Invitrogen, Carlsbad, CA, USA). Reverse transcription reactions were performed with AccuPower RT Premix (Bioneer, Daejeon, Korea) using $1 \mu \mathrm{g}$ total RNA. qRT-PCR was performed with SYBR Premix Ex Taq (Takara Bio, Shiga, Japan) in ABI 7500 real-time PCR system (Applied Biosystems, Waltham, MA, USA) using the specific primers: $\beta$-actin, $5^{\prime}$-TCC TCT CCC AAG TCC ACA CAG G-3' (forward) and 5'-GGG CAC GAA GGC TCA TCA TTC-3' (reverse); MET, 5'-TCA TTG GTT CCA ATC ACA GCT-3' (forward) and 5'-GCC ACC GAG ACA GAG GCT AAT C-3' (reverse); HGF, 5'-CTC ACA CCC GCT GGG AGT AC-3' (forward) and 5'-TCC TTG ACC TTG GAT GCA TTC-3' (reverse). The relative mRNA levels of target genes were determined using 
the comparative $\mathrm{Ct}(\Delta \Delta \mathrm{Ct})$ method and were normalised to the expression levels of $\beta$-actin mRNA.

\section{Western blot analysis}

The cells were either treated with HGF or CAF supernatants for $15 \mathrm{~min}$ in addition to incubation with anti-HGF neutralising antibody or control immunoglobulin $\mathrm{G}(\mathrm{IgG})$ for $1 \mathrm{~h}$ in the experiments. Following incubation, the cells were treated with crizotinib for $1 \mathrm{~h}$. The cells were then washed with PBS and lysed with PRO-PREP protein extraction solution (iNtRON Biotechnology, Seongnam, Gyeonggi-do, South Korea). Lysates were placed on ice for $30 \mathrm{~min}$ and centrifuged at $13,000 \mathrm{rpm}$ for $20 \mathrm{~min}$ at $4{ }^{\circ} \mathrm{C}$. The protein concentration was determined by the Bradford assay (Bio-Rad, Hercules, CA, USA). Equal amounts of protein from each sample were resolved by sodium dodecyl sulfate-polyacrylamide gel electrophoresis (SDS-PAGE) and transferred onto nitrocellulose membranes (EMD Millipore, Billerica, MA, USA). The immunoblots were blocked by treatment with 5\% skim milk, $25 \mathrm{mM}$ Tris- $\mathrm{HCl}$ ( $\mathrm{pH} 8.0$ ), $150 \mathrm{mM} \mathrm{NaCl}$, and $0.1 \%$ Tween 20 for $1 \mathrm{~h}$ at room temperature. The membrane was then incubated with primary antibodies overnight at $4{ }^{\circ} \mathrm{C}$. Protein detection was performed using an ECL detection kit (GE Healthcare, Piscataway, NJ, USA).

\section{Cell viability and proliferation assay}

Tumour cells were seeded at a concentration of $4 \times 10^{3}$ cells per well in 96 -well plates or $1 \times 10^{4}$ cells per well in 24-well plates and incubated at $37{ }^{\circ} \mathrm{C}$. Following overnight incubation, the culture media were changed to RPMI 1640 supplemented with $1 \%$ foetal bovine serum for $24 \mathrm{~h}$. The following day, recombinant human HGF, CAF supernatant, crizotinib, and anti-HGF neutralising antibody or control IgG were added to each well at the indicated concentrations, and the cell culture was maintained for $48 \mathrm{~h}$. The cell viability/proliferation assay was performed using the EZ-Cytox assay kit (Daeil Lab Service, Seoul, Korea). The absorbance of the samples was measured using a Synergy ${ }^{\mathrm{TM}} \mathrm{H} 1$ Hybrid Multi-Mode reader (BioTek Instruments, Inc., Winooski, VT, USA) at $450 \mathrm{~nm}$. All experiments were performed in triplicates.

\section{Enzyme-linked immunosorbent assay (ELISA)}

The concentration of human HGF in the supernatants of GC cells and CAF was calculated using the human HGF Quantikine ELISA kit (R\&D Systems) according to the manufacturer's instructions. Briefly, the cells were cultured in 100mm culture dishes with RPMI 1640 supplemented with 1\% foetal bovine serum, and the culture media were changed at the time of achieving $80 \%$ cellular confluence. At $72 \mathrm{~h}$ after the media change, the supernatants (conditioned media, CM) were harvested for ELISA. The serially diluted supernatants ( $20 \%, 2 \%$ and $0.2 \%$ by adding RPMI media) were used for the experiments.

\section{Transwell invasion assay}

In vitro Matrigel invasion assays were performed using 24-well Boyden chamber $(8.0 \mu \mathrm{m}$ pore size, Corning, New York, NY, USA). The Transwell filters were coated with the appropriate Matrigel (3 mg/mL; Becton Dickison, Franklin Lakes, NJ, USA), and $1 \times 10^{5}$ cells were seeded onto the Matrigel following solidification at $37{ }^{\circ} \mathrm{C}$. After $48 \mathrm{~h}$, the cells that invaded the Matrigel and were attached to the lower surface of the filter were fixed with methanol and stained with haematoxylin and eosin. The stained cells were visualised and photographed under an inverted bright-field microscope at $200 \times$ magnification. We chose random fields in the chamber to count the cells using Image J software. All experiments were performed in triplicates.

\section{Immunohistochemistry (IHC)}

The IHC study was carried out on 4- $\mu$ m-thick, FFPE tissue sections using an automated immunostainer (Ventana Medical System) according to the manufacturer's instructions. The primary antibodies included anti-total MET (SP44) rabbit monoclonal antibody (Ventana Medical System, Tucson, AZ, USA) and anti-phospho (p)-MET (Y1349) rabbit monoclonal antibody (Abcam, Cambridge, MA, USA). Two independent pathologists ( $\mathrm{SK}$ and JHH) blindly evaluated the stained slides. As SP44 antibody is directed against a membranous and/or cytoplasmic epitope present in human normal epithelial or tumour cells, we adopted an interpretation method based on both membranous and/or cytoplasmic staining [19]. The staining intensity was scored as follows: 0 , no reactivity or faint staining; +1 , faint or weak staining; +2 , moderate staining; +3 , strong staining. MET positivity was defined as $\geq 25 \%$ of tumour cells with $\geq+1$ staining intensity, as previously suggested [15]. As for p-MET, tumour samples were interpreted as p-MET positive when $\geq 50 \%$ of cancer cells revealed staining intensity $\geq+2$, as suggested [20].

\section{Gene set enrichment analysis (GSEA)}

The expression dataset of 151 GC patients (GSE15459) [21] was obtained from the GEO database (www.ncbi.nlm. nih.gov/geo). GSEA was performed using the cloud version of GSEA, version 19.0.25 (https://cloud.genepatter n.org) with default parameters [22]. The samples were classified as either MET-positive (50 patients) or METnegative (101 patients) according to the MET expression 
analysed by GSEA with the BIOCARTA_MET_PATHWAY (M19358) gene signature. Among the MET-positive samples, five cases were identified as HGF-positive, and the remaining 45 cases were designated as HGF-negative according to the M19358 gene signature. The gene signatures CELL_PROLIFERATION_GO_0008283 (M1621), CELL_MIGRATION (M6281), MISHRA_CARCINOMA_ASSOCIATED_FIBROBLAST_UP (M18292) and MISHRA_CARCINOMA_ASSOCIATED_FIBROBLAST_ DN (M4577) were used for further analysis.

\section{Statistical analysis}

Statistical analysis was performed using IBM SPSS ver.22 (IBM Corp.) and GraphPad Prism 7.00 (GraphPad Software). Mann-Whitney $U$ test, logistic regression test, Chisquare test, Fisher's exact test, or unpaired Student's $t$ test were used as deemed appropriate. Survival analyses were performed using the Kaplan-Meier method and log-rank test. All experimental data are expressed as means $\pm \mathrm{SD}$ (standard deviation). A $p$ value $<0.05$ is considered statistically significant. All reported $p$ values are two-sided.

\section{Results}

\section{Ligand-independent MET expression does not ensure MET signalling activation}

For more than a decade, investigators have assumed that MET protein overexpression without $M E T$ amplification is another sign for MET signalling activation; thus, it has been regarded as an indicator for therapeutics targeting the HGFMET axis [15-17]. Strong IHC staining for MET (score + 3) has been correlated with $M E T$ amplification [23], whereas it remains unknown whether low to intermediate intensity of staining (score +1 and +2 ) represent a spectrum of MET signalling activation in cancer tissue. To answer this, we screened MET mRNA and protein levels in $11 \mathrm{GC}$ cell lines using qRT-PCR and western blotting, respectively (Fig. 1a, b). We found that MET expression was diverse across the cell lines, whereas their mRNA and protein expressions were relatively well correlated. Among these, the known METamplified cell line MKN45 showed the highest mRNA and protein expression. Furthermore, phosphorylation of MET, an indicator of MET activation, was clearly observed only in MKN45 cells. In other cell lines, p-MET was almost undetectable, even when the MET protein expression was relatively high, as seen in KATOIII, SNU216, and SNU668. This indicates that low to intermediate grades of MET expression themselves do not indicate MET activation in cancer cells.

Next, we asked whether sufficient HGF can trigger MET signalling activation even in low MET-expressing cancer cells. To answer this, we chose relatively low METexpressing MKN74 and NUGC-4 based on western blotting and treated these cells with serially increasing doses of HGF (from 0.1 to $50 \mathrm{ng} / \mathrm{mL}$ ). This revealed that abundant ( $\geq 10 \mathrm{ng} / \mathrm{mL}$ ) HGF induced MET signalling activation, showing much increased phosphorylation of ERK and AKT (Fig. 1c). In comparison, MET-amplified MKN45 did not show any change in these proteins, meaning that MET
Fig. 1 MET signalling activation in MET-unamplified gastric cancers (GCs) is dependent on the exogenous hepatocyte growth factor (HGF) in a dosedependent manner. a qRT-PCR results of MET mRNA expression levels in GC cell lines. b MET and phosphorylated MET (p-MET) level in GC cell lines by western blot. $\mathbf{c}$ Induction of MET and its downstream pathway components in lowMET expressing MKN74 and NUGC-4 GC cells compared to $M E T$-amplified MKN45 and MET-lacking SNU1 cell lines, according to the serially increasing dose of $\mathrm{HGF}$ $(0.1-50 \mathrm{ng} / \mathrm{mL})$ a

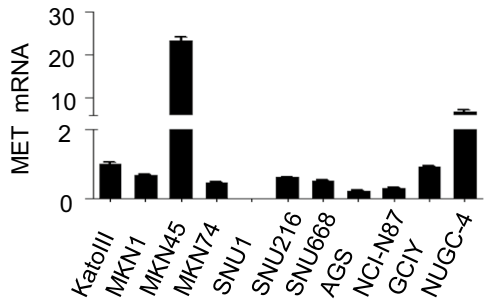

c

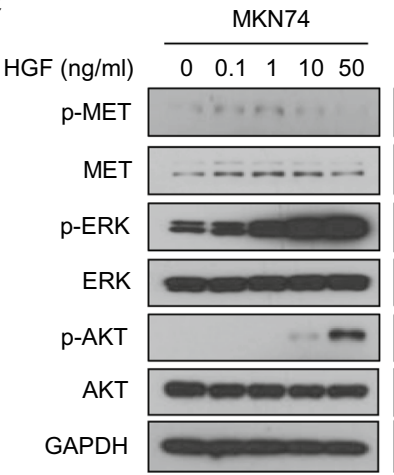

b
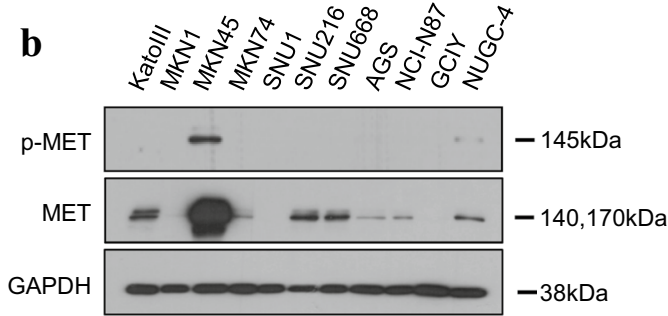

NUGC-4

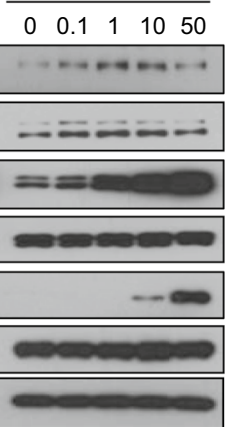

MKN45

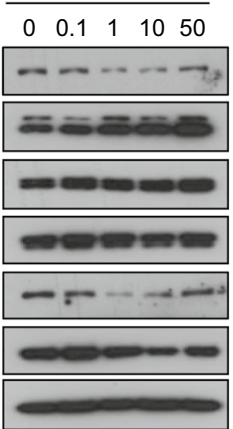

SNU1

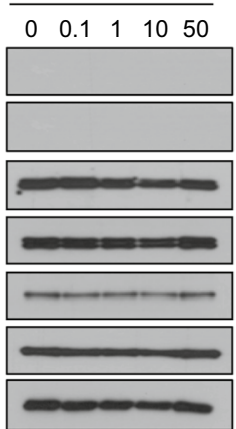


signalling is already sufficiently activated regardless of the exogenous HGF. HGF did not induce the phosphorylation of MET and its downstream nodes even upon high doses of HGF in SNU1, which is devoid of endogenous MET expression. Taken together, these results indicate that sufficient exogenous HGF can fully induce MET signalling activation even in low MET-expressing cancer cells but not in cells with utterly no MET expression.

\section{Abundant HGF induces malignant phenotypes even in cancer cells with low MET expression}

To investigate whether the observed MET signalling activation by HGF is associated with alterations in phenotypes in cancer cells, we performed cell viability and transwell invasion assays. Low MET-expressing MKN74 and NUGC-4 exhibited increased proliferation in response to HGF treatment $(\geq 1 \mathrm{ng} / \mathrm{mL})$ in a dose-dependent manner (Fig. 2a, b). This increased proliferation was efficiently inhibited by the anti-HGF neutralising antibody (Fig. 2c, d). In contrast, the viabilities of MKN45 and SNU1 were not affected by exogenous HGF (Fig. 2e, f). High doses of HGF ( $\geq 10 \mathrm{ng} / \mathrm{mL}$ ) also significantly facilitated the cellular invasion of MKN74 and NUGC-1 (Fig. 2g), whereas the invasive properties of MKN45 and SNU1 remained the same regardless of HGF treatment (Supplementary Fig. 1). These results demonstrate that low MET-expressing cancer cells may acquire malignant phenotypes in an HGF-rich environment. However, $M E T$-amplified or MET-lacking cancer cells were not influenced by exogenous HGF.
Fig. 2 Low-MET expressing gastric cancers (GCs) can obtain malignant phenotypes especially upon the high dose of hepatocyte growth factor (HGF) treatment. a, b Cell proliferation assay in response to various doses of exogenous HGF in low MET-expressing MKN74 and NUGC-4 cells. c, d Cell proliferation assay with the treatment of low dose HGF $(0.1 \mathrm{ng} / \mathrm{mL})$ and high dose HGF $(50 \mathrm{ng} / \mathrm{mL})$ in the MKN74 and NUGC-4. Anti-HGF neutralizing antibody $(20 \mu \mathrm{g} / \mathrm{mL})$ was used to block the effect of HGF. e, f Cell viability assay in $M E T$-amplified MKN45 and MET-lacking SNU1 upon exogenous HGF treatment. g Transwell invasion assay for MKN74, NUGC-4, MKN45, and SNU-1 with the treatment of various doses of HGF (0.1-50 ng/mL). Scale bar $=100 \mu \mathrm{m}$. All experiments were performed in triplicate. The results are shown as the mean \pm standard deviation (SD), ${ }^{* *} p<0.01,{ }^{*} p<0.05$ a

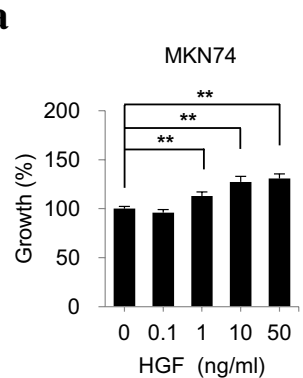

b

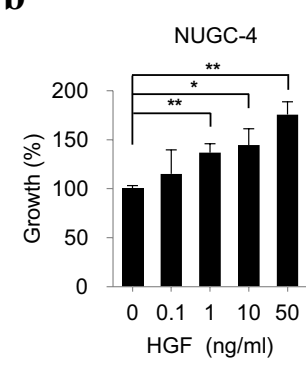

d

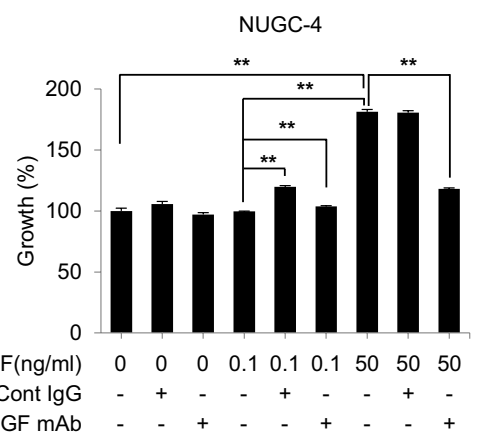

c

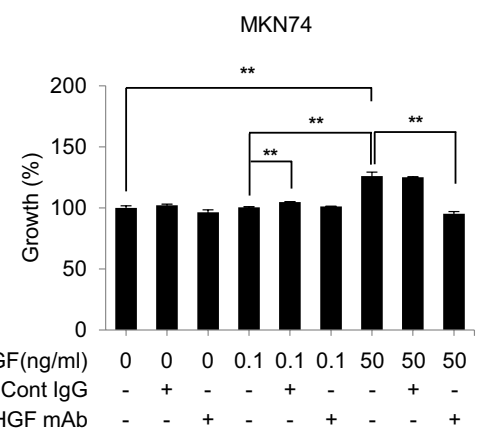

e

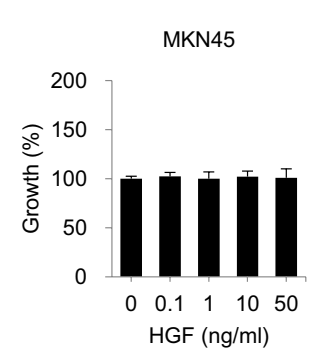

f

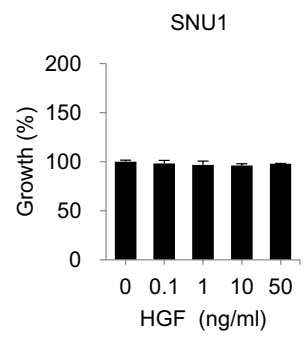

g

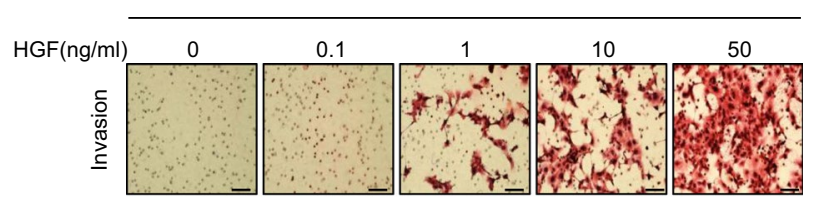

NUGC-4

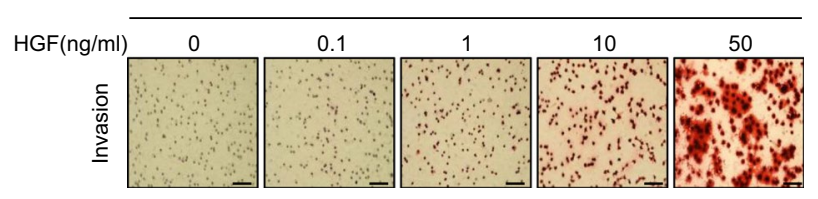

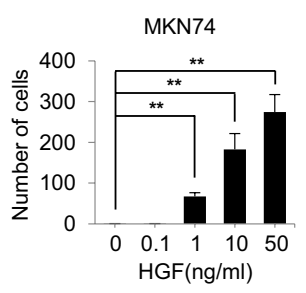

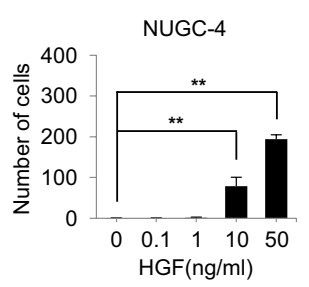




\section{High concentrations of HGF from CAFs foster cancer cell proliferation and invasion through the activation of the MET signalling cascade}

Previous studies have identified that CAFs produce abundant HGF to support tumour growth in a paracrine manner [24-27]. In line with this, to pursue the major source of HGF in the GC microenvironment, we assessed the HGF mRNA levels of CAFs isolated from GC tissue and GC cell lines, and it showed substantially higher levels of HGF in CAFs than in GC cells (Supplementary Fig. 2a). Accordingly, a much greater amount of HGF, approximately 100fold of that of cancer cells, was secreted from CAFs (Supplementary Fig. 2b). This indicates that HGF in the tumour microenvironment may mostly come from CAFs, and it also suggests that the number of CAFs accumulated in the tumour tissue may determine the amount of HGF in the GC microenvironment.

Therefore, we assumed that serially diluted CM from CAFs (100-0.2\%) came from serially decreasing numbers of CAFs. Then, we assessed the amount of the CAF-derived $\mathrm{CM}$ that drives malignant phenotypes in low-MET expressing cancer cells through the activation of MET signalling. Both MKN74 and NUGC-4 exhibited increased cellular proliferation upon treatment with high-concentrated $\mathrm{CM}$ from CAFs (Fig. 3a, b), and these effects were somewhat variably inhibited by anti-HGF antibody, probably due to the different nature of each cell (Fig. 3c, d). Invasive properties of MKN74 and NUGC-4 were much more enhanced when they were treated with high-concentrated CM than with low-concentrated CM, whereas this increased cellular invasion was efficiently inhibited by anti-HGF antibody (Fig. 3e, f, Supplementary Fig. 3). Mechanistically, high concentration of CM only activated MET and its downstream ERK and AKT (Fig. 3g, h). Treatment with anti-HGF antibody reduced the phosphorylation of MET, ERK, and AKT in these cells. On the other hand, MET-amplified MKN45 and MET-lacking SNU1 did not demonstrate any phenotypic changes (Supplementary Fig. 4a, b) as well as signalling alterations (Supplementary Fig. 4c) even with the high-concentrated CM treatment, as expected. Collectively, these results imply that high numbers of CAFs can trigger malignant phenotypes of cancer by secreting sufficient amounts of HGF, even in lowMET expressing cancer cells.

\section{High stromal proportion denotes worse patient survival only in MET-expressing GCs}

To investigate the clinical relevance of our in vitro data, we assumed that a high number of CAFs corresponds to a high stromal proportion in cancer tissue, as previously suggested [18]. Thus, we analysed the stromal proportions of
272 advanced GC tissues and classified them into stromalow $(<50 \%)$ and stroma-high $(\geq 50 \%)$ (Fig. $4 \mathrm{a})$, as previously indicated [18]. Then, we performed IHC for MET on these samples and deemed all MET-expressing cancers as positive regardless of the intensity (Fig. 4b). In our cohort, MET expression (MET-positivity) did not impact patient prognosis (Fig. 4c). Even if we considered only $2 / 3$ intensity as positive, MET-positivity itself did not influence patient survival (data not shown). However, a high stromal proportion indicated worse prognosis (Fig. 4d), in line with many previous studies [18, 28].

Therefore, we aimed to evaluate the stromal effect according to MET expression, and re-analysed the survival of the patients with regard to the stromal proportion in MET-positive and MET-negative cancers, separately. Surprisingly, a high stromal proportion was associated with a much worse prognosis in patients with MET-positive GCs $(p=0.001)$, whereas stromal proportion did not impact survival in METnegative tumours ( $p=0.361)$ (Fig. $4 \mathrm{e})$. This strongly suggests that a high volume of stroma (i.e., high number of CAFs and abundant HGF) induces sufficient MET signalling activation even in low MET-expressing tumours. However, a large volume of stromal CAFs could not trigger MET signalling activation in MET-lacking tumours, similar to the results of our experimental data. As a result, a high stromal proportion was meaningless in MET-negative tumours. Because the majority of 'stroma-high' tumours were histologically poorly cohesive carcinomas, which exhibit profuse fibrosis and dismal prognosis, we performed the same analysis using only poorly cohesive carcinomas from our cohort. We found that the previously identified stromal effect was even more accentuated in MET-positive poorly cohesive carcinomas $(p<0.001)$, whereas a high stromal proportion had no effect on prognosis in MET-negative tumours $(p=0.647)$ (Fig. 4f). For further validation, we performed IHC for p-MET in MET-positive FFPE samples, although p-MET has very short half-like and its assessment is usually considered not much reliable especially in FFPE tissues by IHC as stated previously [29]. Higher proportion of p-MET positivity was observed in stroma-high GCs compared to stroma-low cases $(21.7 \%$ vs. $9.17 \%, p=0.0174$; Supplementary Fig. 5).

In support of our data, the GSEA from the public database (GSE15459) [21] revealed that the MET pathway signature was enriched in MET-positive samples when HGF was high (Fig. 5a). In line with this, the gene sets regarding cell proliferation, cell migration, and CAF were also enriched in simultaneously MET- and HGF-positive samples (Fig. 5b-d). Taken together, these data suggest that abundant stroma (sufficient HGF)-induced MET signalling activation is much more important for determining the clinical outcome of patients than MET expression itself in cancer cells. Moreover, it also suggests that MET-positivity (even with 


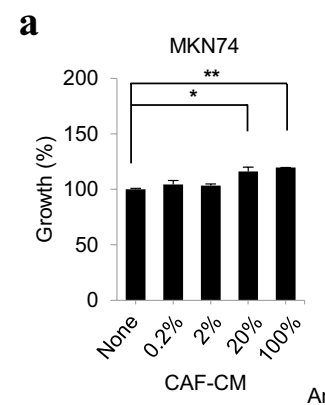

c

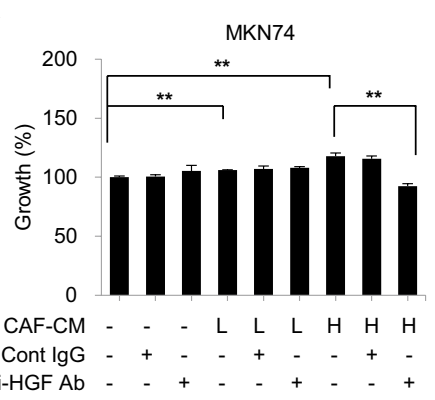

e

MKN74
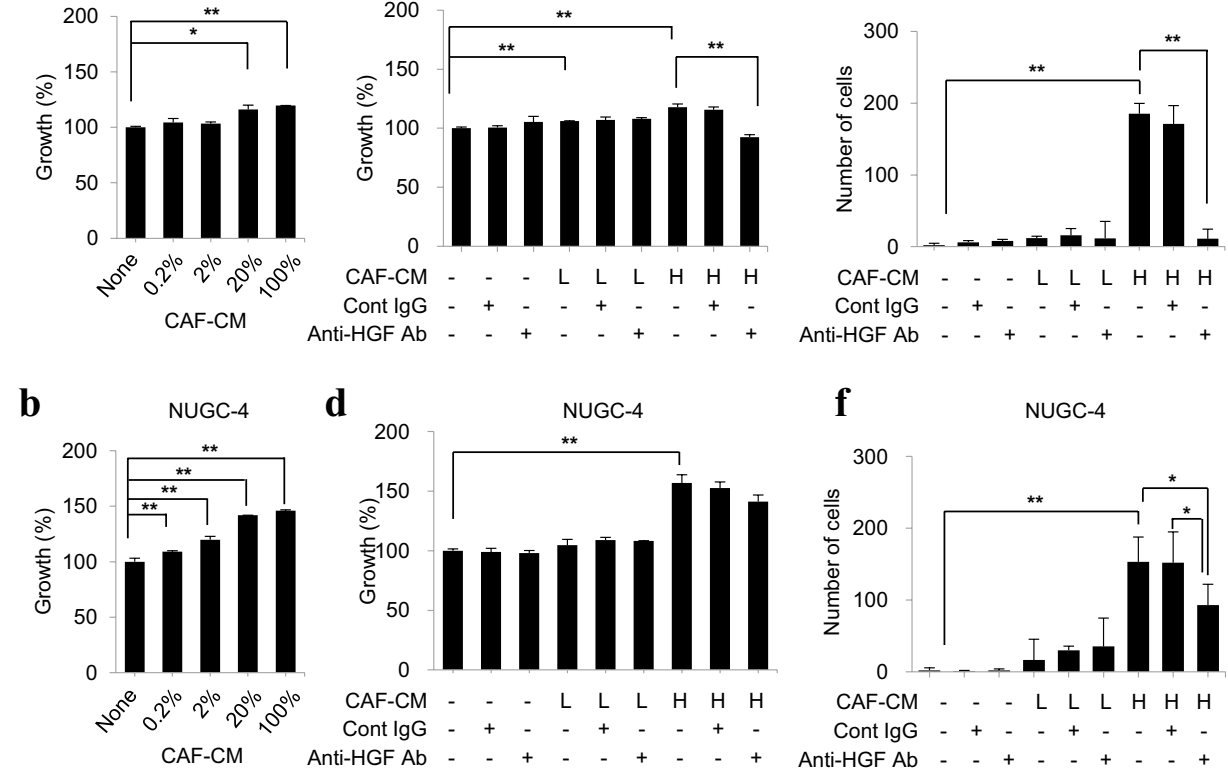

g
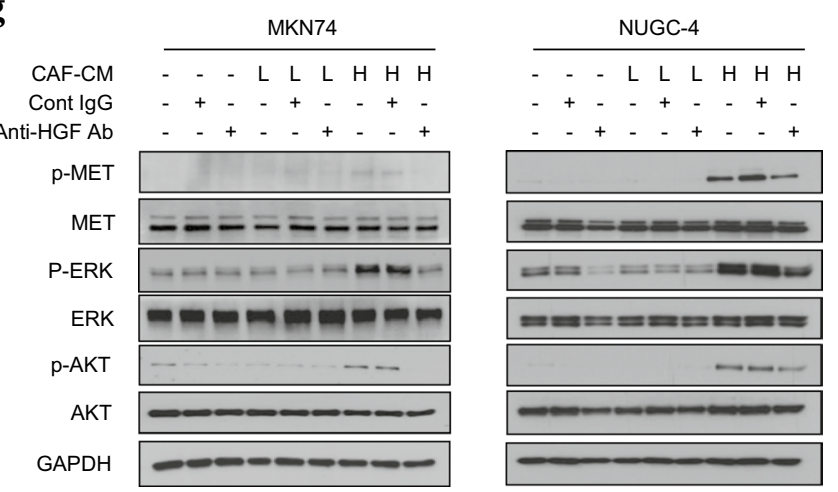

Fig. 3 High-concentrated conditioned media (CM) from cancer-associated fibroblasts (CAFs) induce sufficient MET signalling activation and corresponding phenotypical changes even in low-MET expressing cancer cells. a, b Proliferation of low-MET expressing MKN74 and NUGC-4 GC cell lines in response to serially diluted CM from CAFs $(0.2 \%, 1 / 500$ diluted CM; 2\%, 1/50 diluted; 20\%, 1/5 diluted; $100 \%$, non-diluted). CM was obtained after $72 \mathrm{~h}$ incubation of CAFs. The cell count was $7.5 \times 10^{4}$ at the time of harvest. c, d Suppressed tumour cell growth by anti-hepatocyte growth factor (HGF) neutralising antibody $(20 \mu \mathrm{g} / \mathrm{mL})$ in MKN74 (c) and NUGC-4 (d) in the pres-

a weak intensity) and sufficient HGF (practically, a large amount of stroma) may be two essential elements for better prediction of MET-signalling activity in clinical samples.

\section{Ligand-dependent MET signalling activation enhances the response to MET-targeted therapy}

We hypothesised that sufficient HGF-induced MET activation may be a prerequisite for improved antitumor activity of an anti-MET therapy even in low-MET expressing cancers. To prove this, first, as a control, we treated MET ence of CM from CAF. e, $\mathbf{f}$ Transwell invasion assay for MKN74 (e) and NUGC-4 (f) with the treatment of CM from CAF (L, low concentration of $\mathrm{CM}, 0.2 \%$; $\mathrm{H}$, high concentration, 100\%). $\mathrm{g}$ Induction of MET downstream pathway components in MKN74 and NUGC-4 by $\mathrm{CM}$ from $\mathrm{CAF}$ ( $L$ low concentration of $\mathrm{CM}, 0.2 \%$; $H$ high concentration, $100 \%)$ and their blockage by anti-HGF neutralising antibody $(20 \mu \mathrm{g} / \mathrm{mL})$. All experiments were performed in triplicate. The results are shown as the mean \pm standard deviation $(\mathrm{SD}),{ }^{*} p<0.01$, $* p<0.05$

tyrosine kinase inhibitor crizotinib on MKN45, which demonstrated vulnerability to crizotinib regardless of HGF co-treatment (IC50 $=0.0429$ and 0.0526 for $0 \mathrm{ng} / \mathrm{mL}$ and $50 \mathrm{ng} / \mathrm{mL}$ of HGF treatment, respectively) (Fig. 6a). Without HGF, MKN74 and NUGC-4 were resistant to crizotinib (IC50 $=3.78$ for MKN74 and 4.27 for NUGC-4). However, HGF-treated MKN74 and NUGC-4 showed dramatically increased sensitivities upon crizotinib treatment (IC50 = 2.62 for MKN74 and 0.894 for NUGC-4) (Fig. 6b, c). In detail, without HGF, the growth inhibitory effects of crizotinib on MKN74 and NUGC-4 were much lower than 
$\mathbf{a}$

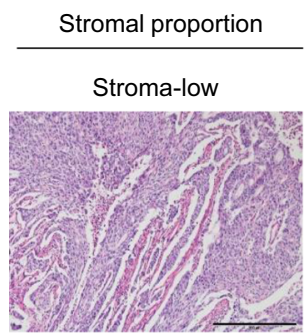

Stroma-high

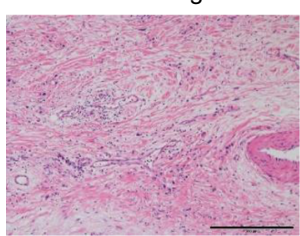

b

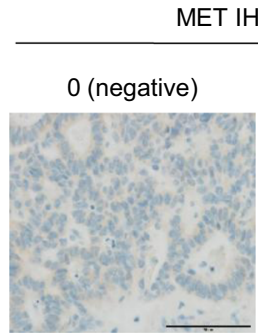

+2 (positive)

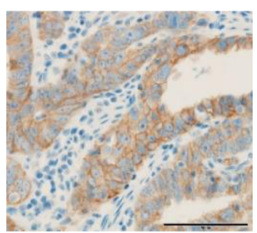

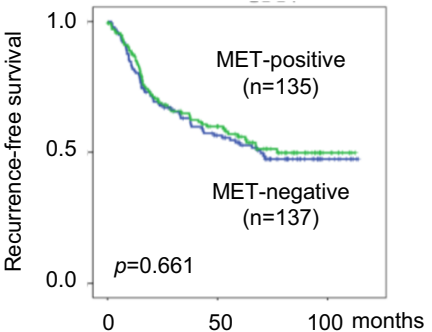

d

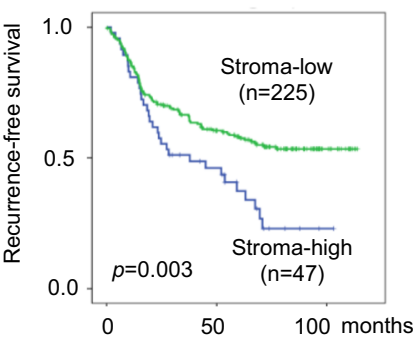

$\mathbf{e}$

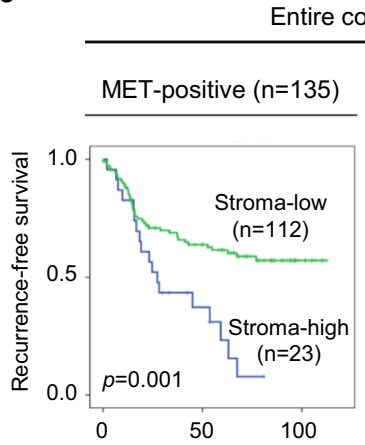

Entire cohort $(n=272)$

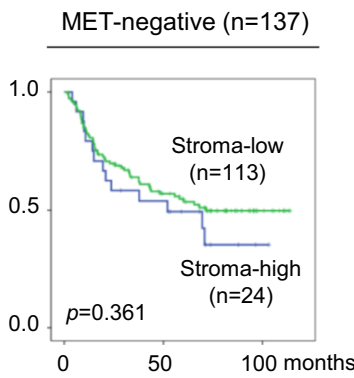

f

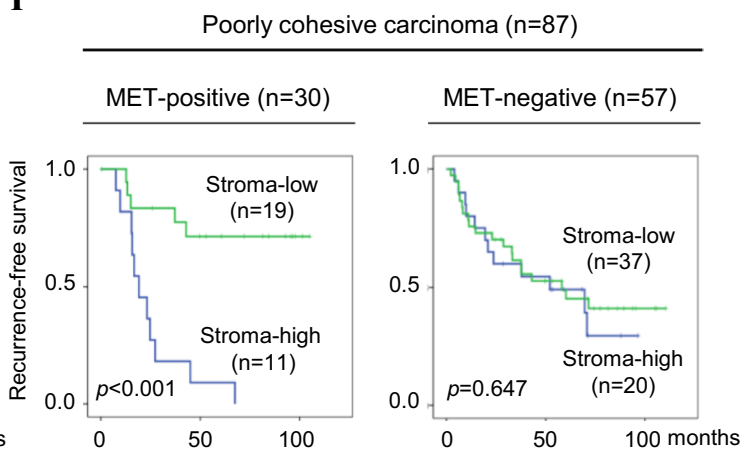

Fig. 4 Prognostic significance of the stromal proportion according to the MET positivity in gastric cancer (GC) tissue. a Representative histological images of low and high stromal components in the cancer tissue. Scale bar $=500 \mu \mathrm{m}$. b Representative immunohistochemical staining images for MET. Scale bar $=100 \mu \mathrm{m}$. c Survival curves for the entire $272 \mathrm{GC}$ patients according to MET expression status.

those on MET-amplified MKN45, whereas high doses of HGF treatment significantly enhanced the drug sensitivities of MKN74 and NUGC-4, and the antitumor effects on these cells were comparable to those on MKN45 (Fig. 6d). As HGF-treated cancer cells had much higher growth rates, we performed the same experiments using each cell line supplemented by $10 \%$ or $20 \% \mathrm{FBS}$ as a control. These revealed that addition of FBS did not sensitize MKN74 and NUGC-4 to crizotinib in spite of its growth-promoting effects, whereas MET-amplified MKN45 showed sensitivity upon crizotinib treatment regardless of FBS supplementation (Supplementary Fig. 6).

Next, we performed the same experiments using CM from CAFs. Compared to MKN45, CM-untreated MKN74 and NUGC-4 were not responsive to crizotinib. Low-concentrated CM slightly increased the drug sensitivity of these cells, whereas high-concentrated CM substantially enhanced d Survival curves of all the patients according to the stromal proportion. e Survival curves according to the stromal proportion in METpositive cases (left) and MET-negative cases (right). f Survival curves according to the stromal proportion in the poorly cohesive carcinoma cases classified as MET-positive (left) or MET-negative (right). $p$ by log-rank test

the drug sensitivity, and the growth inhibitory effects on these cells became similar or even slightly higher than that on METamplified MKN45 (Fig. 6e). In light of signalling, high-concentration CM significantly increased the phosphorylation of MET as well as its downstream ERK and AKT phosphorylation, which were abrogated by crizotinib treatment (Fig. 6f). These collective data imply that MET signalling activation triggered by high concentrations of HGF mainly from CAFs can substantially sensitise the low MET-expressing cancer cells to an anti-MET treatment, just as much as the METamplified cancers. 
Fig. 5 Gene set enrichment analysis on gastric cancer (GC) tissues from the public database (GSE15459). a MET pathway signature. b Cell proliferationrelated signature. c Cell migration-related signature. $\mathbf{d}$ Cancer-associated fibroblastrelated signature. All these cases were MET-positive, and the analysis was done according to the hepatocyte growth factor (HGF) positivity $\mathbf{a}$

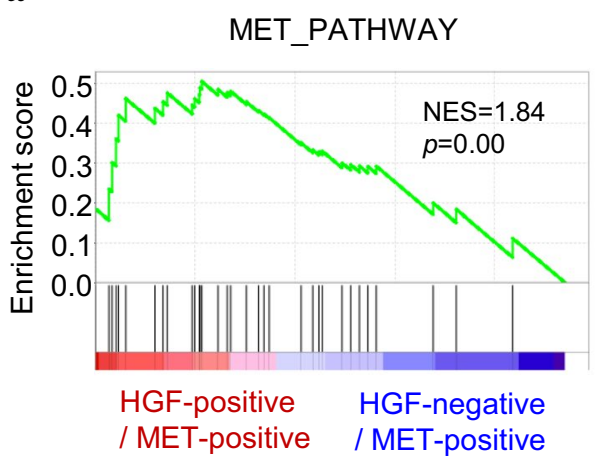

c

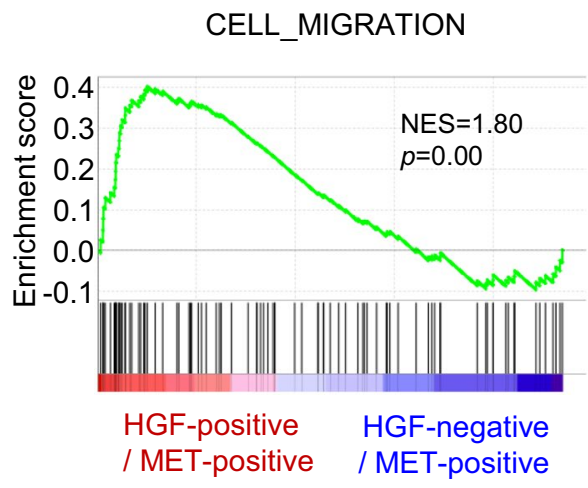

b

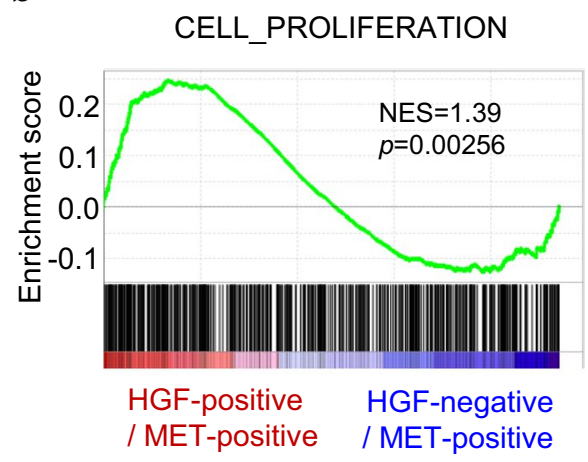

d

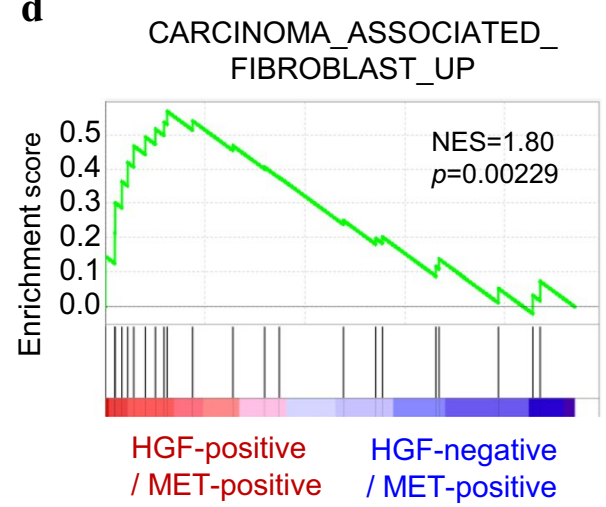

\section{Discussion}

The reason that preclinical success of the strategy targeting the HGF-MET axis was not related to clinical success may be largely attributed to the failure of selecting appropriate patients based on reliable biomarkers. This simply means that preclinical experiments were not translated well into the clinics. In many previous studies, investigators only treated high doses of HGF (commonly $50 \mathrm{ng} / \mathrm{mL}$ ) to activate MET signalling in cancer cells and used this to evaluate the drug response in preclinical models. However, they did not consider the actual amount of HGF collected in the tumour microenvironment. Nonetheless, with the assumption of the presence of sufficient HGF, clinical trials only enrolled patients with MET-expressing cancer [15-17]. Some researchers have investigated serum HGF as an alternative, but it also seems to be unsuccessful [15]. This may be explained by the following reasons: (1) serum HGF may be quite different from the actual HGF concentration in the tumour microenvironment, (2) the cut-off dividing HGFhigh and HGF-low groups was not calculated based on biology but was just determined by the median value, and (3) HGF remains as a biologically inactive precursor form in plasma but is cleaved by serine proteases into biologically active two-chain HGF (tcHGF) in tumor microenvironment [30]. This tcHGC has never been examined in previous clinical trials.
In our study, we conceived a new idea that large amounts of HGF may be derived from an increased number of CAFs in the tumour tissue, which may be collected in tumours with high stromal proportion. We indirectly validated this concept by showing that MET-positive cancers had a much worse prognosis only when the tumours had a high stromal proportion. In line with this, GSEAs demonstrated that the MET-positive GCs were enriched in MET signalling and CAF-related gene signatures when high levels of HGF were present, which underscores the importance of liganddependent MET signalling activation in the clinical samples. Our results also indicate that HGF-dependent MET activation requires at least low MET expression $($ score +1$)$ in cancer cells because MET-negative cancer cells experimentally did not respond to highly concentrated HGF, and MET-negative tumours were not influenced by the stromal proportion in our clinical samples. Previous clinical trials also noted the lack of a positive effect of targeting the MET pathway in patients who were negative for MET expression [31]. Consequently, we demonstrated that HGF-dependent MET signalling is sufficiently activated to be targeted only in a subset of patients, and we propose that this group of patients may be predicted by at least low MET protein expression in cancer cells and high stromal proportion in cancer tissue. To estimate HGF-dependent MET signalling activation in the clinical samples, we firstly introduced a novel concept of stromal proportion as a substitute for HGF 

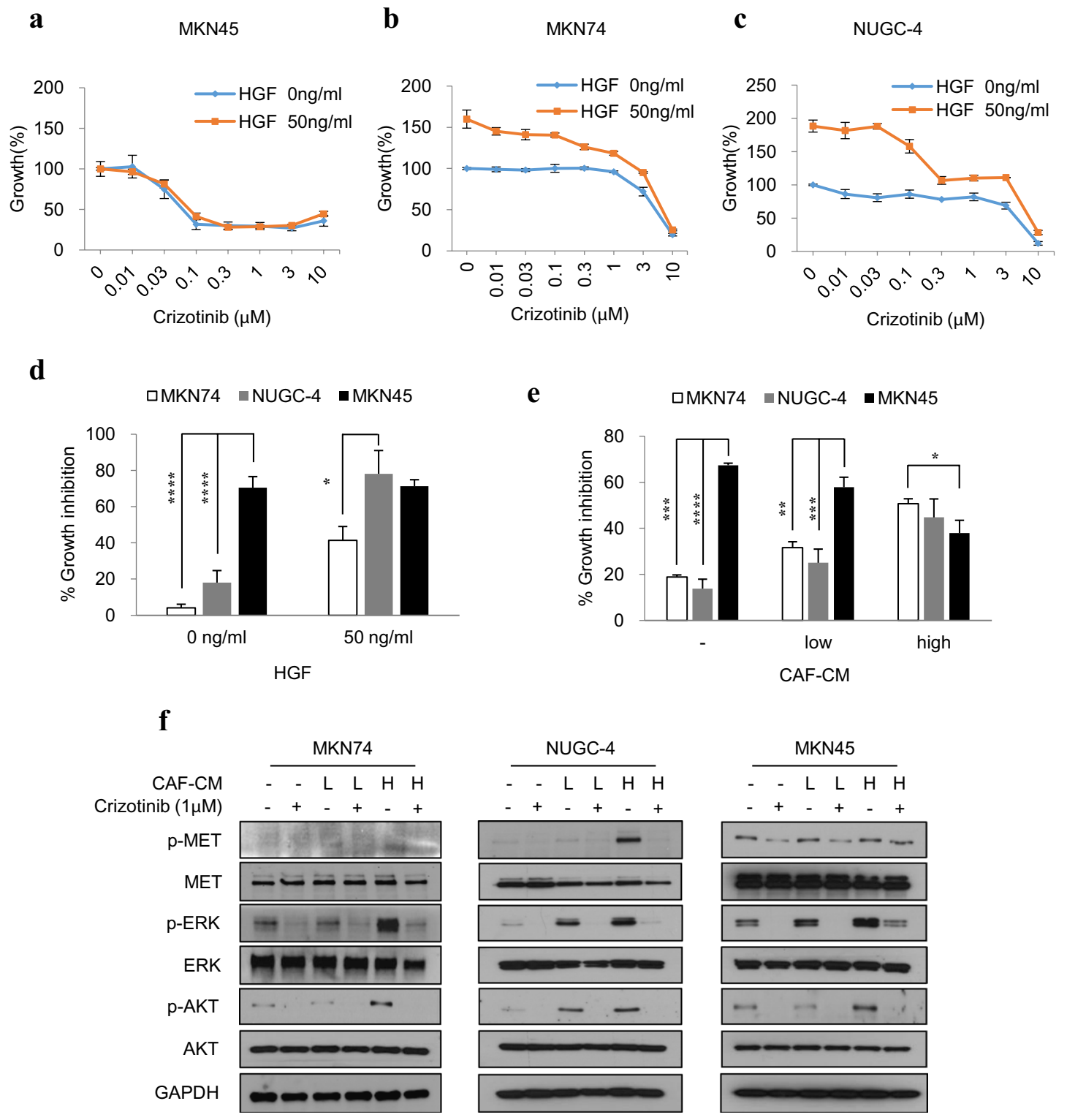

Fig. 6 High-concentrated conditioned media (CM) from cancerassociated fibroblasts (CAFs) significantly enhances the sensitivity to crizotinib in low MET-expressing cancer cells. a-c The growth inhibition of $M E T$-amplified cell line (MKN45, a) and low-MET expressing GC cell lines (MKN74, b; NUGC-4, c) upon the crizotinib treatment with or without hepatocyte growth factor (HGF) treatment. d, e The level of suppressed proliferation of MKN74, NUGC-4,

concentration in tumour tissue, and this would be a monumental achievement especially from a clinical point of view.

MET expression has been found more frequently in intestinal-type than diffuse-type GC [19, 32]. In our study, MET expression was observed in 58.9\% of intestinal-type cancers, whereas only $38 \%$ of diffuse-type cancers were MET-positive $(p=0.001)$ (Table S2). As almost all clinical trials have enrolled patients based on MET expression in cancer tissue, more intestinal-type cancer patients have and MKN45 at $1 \mu \mathrm{M}$ concentration of crizotinib upon the treatment of HGF (d) or CM from CAF (e). $\mathbf{f}$ The inhibition of CAF-mediated MET pathway activation by the treatment of crizotinib ( $L$ low concentration of $\mathrm{CM}, 0.2 \%$; $H$ high concentration, $100 \%$ ). All experiments were performed in triplicate. The results are shown as the mean \pm standard deviation (SD), $* * p<0.01$

been enrolled than patients with diffuse-type cancer. In contrast, a high stromal proportion was much more frequent in diffuse-type cancers than in intestinal-type cancers $(p<0.001)$ (Table S3). In particular, poorly cohesive carcinomas constituted the majority of stroma-high cases $(65.9 \%, p<0.001)$, whereas their MET expression was the lowest among different histologies (Table S4). Based on our results, as MET-expressing cancer with high stromal proportion is expected to respond well to a MET-targeted 
therapy, it is suggested that case selection in previous clinical trials might not be appropriate from our perspective, and diffuse-type GC rather could benefit more from this targeted therapy. Interestingly, higher stromal proportion and enrichment of stromal gene signatures were consistently found much more frequently in female patients [18, 33]. In support of our data, a previous phase I study, which assessed onartuzumab-inhibits HGF binding and subsequent receptor activation-had reported the first complete and durable response in a female patient with GC, whose histology was poorly differentiated adenocarcinoma with signet ring cell features [34].

Although a number of studies have demonstrated that MET expression is associated with advanced stage and shortened survival $[14,32,35]$, we could not identify the prognostic impact of MET expression in our samples. In fact, the reported range of MET expression (24-74\%) is quite wide [11-14]. This variability may stem from the fact that each study depended on different cohort selection, antibody (monoclonal vs. polyclonal), staining procedure, and notably, interpretation and scoring standard of the IHC result. Thus, in line with our study, there are still conflicting data showing that MET expression has no prognostic relevance $[15,36]$, regardless of how they change their interpretation method.

In spite of our unique approaches, we still could not calculate exact amount of HGF in tumor microenvironment. In our study, we just estimated the amount of HGF using stromal proportion. However, even if each tumor has same stromal proportion, the number of CAFs may be quite different in the stroma. Moreover, the amount of secreted HGF also may be different according to the CAFs of each case. Recently, Sakai et al. discovered HiP-8, a small macrocyclic peptide, which selectively binds to tcHGF, and demonstrated that selective detection of tcHGF by Hip-8 provided more precise evaluation of MET activation status in human cancer tissue [37]. This new innovative molecular tool may improve the outcomes of future clinical trials targeting HGF/MET axis.

In conclusion, for the first time, we introduced a new concept regarding the amount of HGF in the tumour microenvironment that can1 fully activate MET signalling in cancer cells. We also connected this concept: the amount of HGF, sequentially to the number of CAFs and finally to the stromal proportion in cancer tissue. Based on these results, we proved that HGF-dependent MET signalling activation may be evident in only a subset of patients with GC, whose tumour has a high stromal proportion and at least low MET expression. We propose that this subset of patients would benefit more from MET-targeted therapy. We hope that our approach will help other researchers to be one step closer to the clinical success of the therapeutic treatment of GC.
Acknowledgements We thank Dr. Seongyeol Park (Korea Advanced Institute of Science and Technology) for advice on performing and analysing Gene Set Enrichment Analysis.

Author contributions DL designed the study and supervised the entire experimental process. SK and DL summarised clinical information and carried out a pathologic slide review. JMA and WJB performed in vitro experiments. SK and JHH and examined the IHC slides. DL conducted the survival analysis. SK performed the gene set enrichment analysis. SK and DL wrote the manuscript. All authors were involved in the critical review and discussion.

Funding This research was supported by the Basic Science Research Programs through the National Research Foundation of Korea (NRF), funded by the Ministry of Science and ICT (2017R1C1B2003970) and by the Ministry of Education (2018R1D1A1B07048078). This research was also supported by the New Faculty Research Fund of Ajou University School of Medicine.

Data availability The data used and/or analysed in this study are available from the corresponding author upon reasonable request.

\section{Compliance with ethical standards}

Conflict of interest The authors declare no conflict of interest.

Ethics approval and consent to participate This study protocol was performed in accordance with the code of ethics of the World Medical Association (Declaration of Helsinki) and was approved by the Institutional Review Board of the Ajou University Hospital (AJIRBBMR-KSR-15-085).

Consent for publication Not applicable.

\section{References}

1. Gherardi E, Birchmeier W, Birchmeier C, Vande WG. Targeting MET in cancer: rationale and progress. Nat Rev Cancer. 2012;12:89-103.

2. Paumelle R, Tulasne D, Kherrouche Z, Plaza S, Leroy C, Reveneau $S$, et al. Hepatocyte growth factor/scatter factor activates the ETS1 transcription factor by a RAS-RAF-MEK-ERK signaling pathway. Oncogene. 2002;21:2309-19.

3. Corso S, Comoglio PM, Giordano S. Cancer therapy: can the challenge be MET? Trends Mol Med. 2005;11:284-92.

4. Kawakami H, Okamoto I. MET-targeted therapy for gastric cancer: the importance of a biomarker-based strategy. Gastric Cancer. 2016;19:687-95.

5. Gavine PR, Ren Y, Han L, Lv J, Fan S, Zhang W, et al. Volitinib, a potent and highly selective c-Met inhibitor, effectively blocks c-Met signaling and growth in c-MET amplified gastric cancer patient-derived tumor xenograft models. Mol Oncol. 2015;9:323-33.

6. Lennerz JK, Kwak EL, Ackerman A, Michael M, Fox SB, Bergethon K, et al. MET amplification identifies a small and aggressive subgroup of esophagogastric adenocarcinoma with evidence of responsiveness to crizotinib. J Clin Oncol. 2011;29:4803-10.

7. Liu YJ, Shen D, Yin X, Gavine P, Zhang T, Su X, et al. HER2, MET and FGFR2 oncogenic driver alterations define distinct molecular segments for targeted therapies in gastric carcinoma. Br J Cancer. 2014;110:1169-78. 
8. Okamoto W, Okamoto I, Arao T, Kuwata K, Hatashita E, Yamaguchi $\mathrm{H}$, et al. Antitumor action of the MET tyrosine kinase inhibitor crizotinib (PF-02341066) in gastric cancer positive for MET amplification. Mol Cancer Ther. 2012;11:1557-64.

9. Smolen GA, Sordella R, Muir B, Mohapatra G, Barmettler A, Archibald $\mathrm{H}$, et al. Amplification of MET may identify a subset of cancers with extreme sensitivity to the selective tyrosine kinase inhibitor PHA-665752. Proc Natl Acad Sci USA. 2006;103:2316-21.

10. Liu L, Zeng W, Wortinger MA, Yan SB, Cornwell P, Peek VL, et al. LY2875358, a neutralizing and internalizing anti-MET bivalent antibody, inhibits HGF-dependent and HGF-independent MET activation and tumor growth. Clin Cancer Res. 2014;20:6059-70.

11. Catenacci DV, Cervantes G, Yala S, Nelson EA, El-Hashani E, Kanteti R, et al. RON (MST1R) is a novel prognostic marker and therapeutic target for gastroesophageal adenocarcinoma. Cancer Biol Ther. 2011;12:9-46.

12. Catenacci DV, Liao WL, Thyparambil S, Henderson L, Xu P, Zhao $\mathrm{L}$, et al. Absolute quantitation of Met using mass spectrometry for clinical application: assay precision, stability, and correlation with MET gene amplification in FFPE tumor tissue. PLoS ONE. 2014;9:e100586.

13. Drebber U, Baldus SE, Nolden B, Grass G, Bollschweiler E, Dienes HP, et al. The overexpression of c-met as a prognostic indicator for gastric carcinoma compared to p53 and p21 nuclear accumulation. Oncol Rep. 2008;19:1477-83.

14. Nakajima M, Sawada H, Yamada Y, Watanabe A, Tatsumi M, Yamashita J, et al. The prognostic significance of amplification and overexpression of c-met and c-erb B-2 in human gastric carcinomas. Cancer. 1999;85:1894-902.

15. Catenacci DVT, Tebbutt NC, Davidenko I, Murad AM, Al-Batran SE, Ilson DH, et al. Rilotumumab plus epirubicin, cisplatin, and capecitabine as first-line therapy in advanced MET-positive gastric or gastro-oesophageal junction cancer (RILOMET-1): a randomised, double-blind, placebo-controlled, phase 3 trial. Lancet Oncol. 2017;18:1467-82.

16. Shah MA, Bang YJ, Lordick F, Alsina M, Chen M, Hack SP, et al. Effect of fluorouracil, leucovorin, and oxaliplatin with or without onartuzumab in HER2-negative, MET-positive gastroesophageal adenocarcinoma: the METGastric randomized clinical trial. JAMA Oncol. 2017;3:620-7.

17. Shah MA, Cho JY, Tan IB, Tebbutt NC, Yen CJ, Kang A, et al. A randomized phase II study of FOLFOX with or without the MET Inhibitor onartuzumab in advanced adenocarcinoma of the stomach and gastroesophageal junction. Oncologist. 2016;21:1085-90.

18. Lee D, Ham IH, Son SY, Han SU, Kim YB, Hur H. Intratumor stromal proportion predicts aggressive phenotype of gastric signet ring cell carcinomas. Gastric Cancer. 2017;20:591-601.

19. Ha SY, Lee J, Kang SY, Do IG, Ahn S, Park JO, et al. MET overexpression assessed by new interpretation method predicts gene amplification and poor survival in advanced gastric carcinomas. Mod Pathol. 2013;26:1632-41.

20. Watermann I, Schmitt B, Stellmacher F, Muller J, Gaber R, Kugler $\mathrm{C}$, et al. Improved diagnostics targeting c-MET in non-small cell lung cancer: expression, amplification and activation? Diagn Pathol. 2015;10:130.

21. Ooi CH, Ivanova T, Wu J, Lee M, Tan IB, Tao J, et al. Oncogenic pathway combinations predict clinical prognosis in gastric cancer. PLoS Genet. 2009;5:e1000676.

22. Subramanian A, Tamayo P, Mootha VK, Mukherjee S, Ebert BL, Gillette MA, et al. Gene set enrichment analysis: a knowledgebased approach for interpreting genome-wide expression profiles. Proc Natl Acad Sci USA. 2005;102:15545-50.

23. Zhang J, Guo L, Liu X, Li W, Ying J. MET overexpression, gene amplification and relevant clinicopathological features in gastric adenocarcinoma. Oncotarget. 2017;8:10264-73.
24. Grugan KD, Miller CG, Yao Y, Michaylira CZ, Ohashi S, KleinSzanto AJ, et al. Fibroblast-secreted hepatocyte growth factor plays a functional role in esophageal squamous cell carcinoma invasion. Proc Natl Acad Sci USA. 2010;107:11026-31.

25. Kanaji N, Yokohira M, Nakano-Narusawa Y, Watanabe N, Imaida $\mathrm{K}$, Kadowaki N, et al. Hepatocyte growth factor produced in lung fibroblasts enhances non-small cell lung cancer cell survival and tumor progression. Respir Res. 2017;18:118.

26. Tyan SW, Kuo WH, Huang CK, Pan CC, Shew JY, Chang KJ, et al. Breast cancer cells induce cancer-associated fibroblasts to secrete hepatocyte growth factor to enhance breast tumorigenesis. PLoS ONE. 2011;6:e15313.

27. Iwazawa T, Shiozaki H, Doki Y, Inoue M, Tamura S, Matsui S, et al. Primary human fibroblasts induce diverse tumor invasiveness: involvement of HGF as an important paracrine factor. Jpn J Cancer Res. 1996;87:1134-42.

28. Kemi N, Eskuri M, Herva A, Leppanen J, Huhta H, Helminen O, et al. Tumour-stroma ratio and prognosis in gastric adenocarcinoma. Br J Cancer. 2018;119:435-9.

29. Rhee H, Kim HY, Choi JH, Woo HG, Yoo JE, Nahm JH, et al. Keratin 19 expression in hepatocellular carcinoma is regulated by fibroblast-derived HGF via a MET-ERK1/2-AP1 and SP1 axis. Cancer Res. 2018;78:1619-31.

30. Naka D, Ishii T, Yoshiyama Y, Miyazawa K, Hara H, Hishida $\mathrm{T}$, et al. Activation of hepatocyte growth factor by proteolytic conversion of a single chain form to a heterodimer. J Biol Chem. 1992;267:20114-9.

31. Iveson T, Donehower RC, Davidenko I, Tjulandin S, Deptala A, Harrison M, et al. Rilotumumab in combination with epirubicin, cisplatin, and capecitabine as first-line treatment for gastric or oesophagogastric junction adenocarcinoma: an open-label, dose de-escalation phase $1 \mathrm{~b}$ study and a double-blind, randomised phase 2 study. Lancet Oncol. 2014;15:1007-18.

32. Janjigian YY, Tang LH, Coit DG, Kelsen DP, Francone TD, Weiser MR, et al. MET expression and amplification in patients with localized gastric cancer. Cancer Epidemiol Biomarkers Prev. 2011;20:1021-7.

33. Wu Y, Grabsch $\mathrm{H}$, Ivanova T, Tan IB, Murray J, Ooi CH, et al. Comprehensive genomic meta-analysis identifies intra-tumoural stroma as a predictor of survival in patients with gastric cancer. Gut. 2013;62:1100-11.

34. Feng Y, Ma PC. Anti-MET targeted therapy has come of age: the first durable complete response with MetMAb in metastatic gastric cancer. Cancer Discov. 2011;1:550-4.

35. Taniguchi K, Yonemura Y, Nojima N, Hirono Y, Fushida S, Fujimura T, et al. The relation between the growth patterns of gastric carcinoma and the expression of hepatocyte growth factor receptor (c-met), autocrine motility factor receptor, and urokinasetype plasminogen activator receptor. Cancer. 1998;82:2112-22.

36. Kang BW, Lee D, Chung HY, Han JH, Kim YB. Tetraspanin CD151 expression associated with prognosis for patients with advanced gastric cancer. J Cancer Res Clin Oncol. 2013; 139:1835-43.

37. Sakai K, Passioura T, Sato H, Ito K, Furuhashi H, Umitsu M, et al. Macrocyclic peptide-based inhibition and imaging of hepatocyte growth factor. Nat Chem Biol. 2019;15:598-606.

Publisher's Note Springer Nature remains neutral with regard to jurisdictional claims in published maps and institutional affiliations. 